\title{
Embedded Multi-Frequency Eddy Current Measurement System for In-Situ Assessment of Metals
}

\author{
Rohan Munjal, Frank Wendler and Olfa Kanoun \\ ${ }^{1}$ Chemnitz University of Technology \\ Professorship for Measurement and Sensor Technology \\ Reichenhainer Straße 70, 09126 Chemnitz \\ rohan.munjal@s2014.tu-chemnitz.de
}

\begin{abstract}
:
In this paper, we propose a novel portable multi-frequency embedded sensor system for eddy current assessment of metal pieces, which is capable to realize in-situ measurements over a wide frequency range. The aim of the sensor system is to measure characteristic changes in conductivity and permeability in regards to working distance between sensor coil and work-piece. Thus, realizing a noncontact in-process measurement with a maximum standard deviation of $0.013 \mu \mathrm{H}$. The obtained data of the electric and magnetic parameters can be used to analyze mechanical state and phase transitions of the material [1].
\end{abstract}

Keywords: Impedance Spectroscopy, Inductance Spectroscopy, Multi-Frequency Measurement, Embedded Sensor System

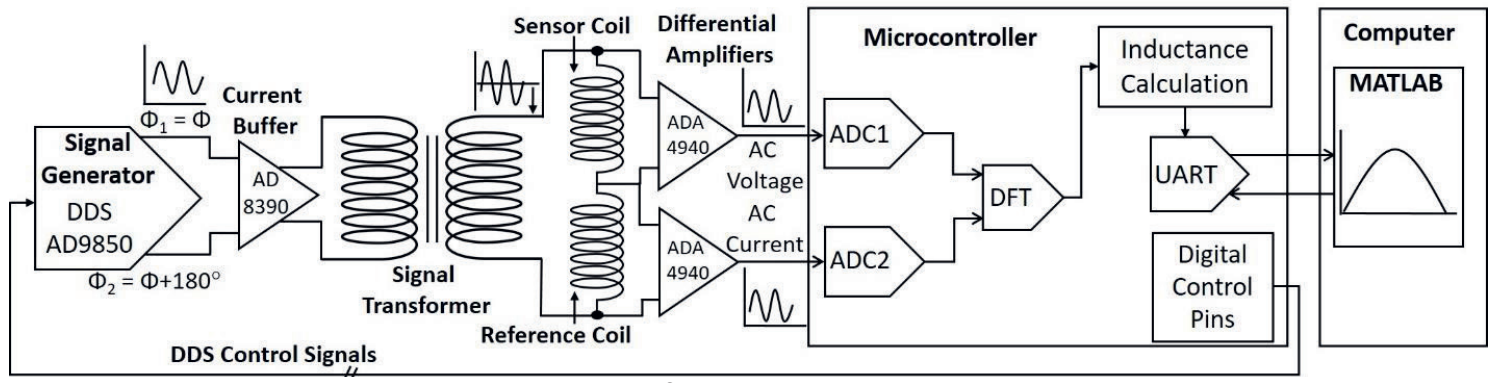

Fig. 1: Block diagram of real-time embedded sensor system

\section{Introduction}

The evolution of manufacturing has led to automization of industry, which requires a more autonomous production processes. Therefore, quality and process control tasks needs sophisticated measurement systems for data acquisition and analysis. In this paper, we discuss the properties of a developed cost effective, portable in-situ, real time and non-contact embedded sensor system, which is targeted for in-process measurements in steel manufacturing.

\section{Embedded Sensor Measurement System}

In [2], we proposed an embedded system for impedance measurement, which can be adopted for the eddy current measurements.

In the Fig. [1], the schematic of the sensor system is shown. The signal generator, current buffer and signal transformer is used to supply the sufficient current for the sensor coil. The current through the sensor coil is calculated in terms of voltage using a reference coil with a similar value as that of sensor coil. The serial combination of sensor and reference coil eliminates the influence of ambient temperature. In this configuration, voltage drop over the both coils are proportional to the individual induct- ance values over the full frequency range. The time domain signal of both coils are conditioned by differential amplifiers and are digitized by inbuilt ADCs of the microcontroller. In the microcontroller DFT is performed to obtained amplitude and phase value of both signals and to calculate the inductance of the sensor coil.

\section{Results}

A partial hardened sample of C-75 steel is used to compare between laboratory device and implemented embedded sensor system.

The unhardened region and hardened region of C-75 steel are measured using impedance analyzer and compared with the simulated Dodd and Deeds model [3]. The obtained results in Fig.[2] show that the unhardened point matches with the relative permeability of 100 and fits to the model simulation, while the hardened point does not matches with the model simulation results and the relative permeability varies between 100 to 125.89 depending upon the frequency as shown in Fig. 2 . The measured inductance from the hardened section is shifted to the simulated model inductance curves with higher permeability values at high frequency around $500 \mathrm{kHz}$. Due to low penetration depth at high frequencies, the obtained information 
from the C-75 steel is confined to the top layer. There is an increase in the variation of permeability in the hardened section as frequency increases, thus higher permeability values are observed at layers close to the metal surface. This is expected from the hardening process as the highest hardening value is expected close to the metal surface, where, highest cooling rate is achieved.

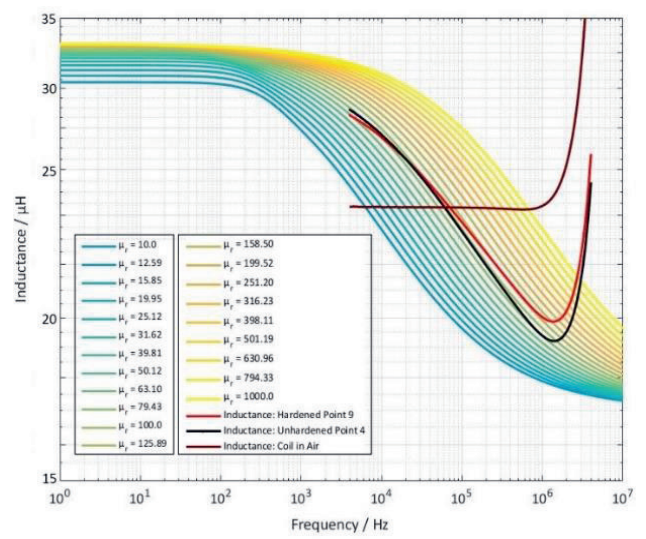

Fig. 2. Model simulation results at logarithmic distributed permeability values and experimental results using impedance analyzer for a C-75 Steel Work Piece

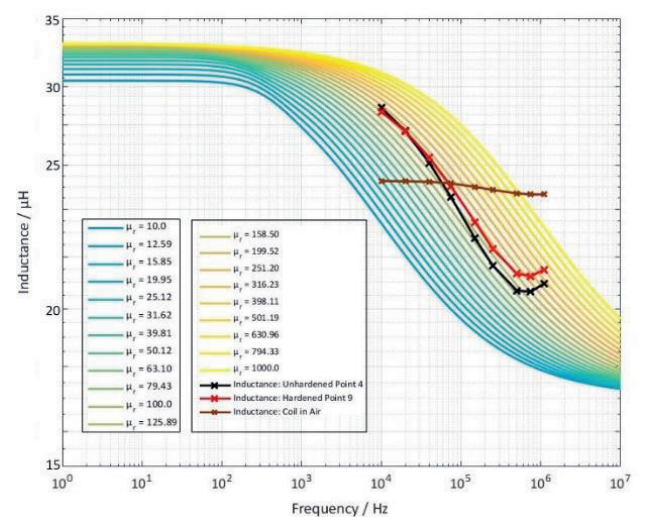

Fig. 3. Model simulation results at logarithmic distributed permeability values and experimental results using embedded sensor system for a C-75 steel work piece

With similar parameters and distance as used in the laboratory measurements, the embedded sensor system measures the inductance of sensor coil with $\mathrm{C}-75$ steel. It shows a higher inductance value in comparison to the measurements on impedance analyzer. Thus, the permeability value for unhardened region fits to 125.89. For the hardened region the permeability varies between 125.89 and 158.50 depending upon the frequency as can be seen in Fig. 3. The obtained higher inductance value from embedded sensor system is due to the presence of uncorrected parasitic inductance on the printed circuit board (PCB), which leads to a constant higher inductance value over the whole frequency range. In the case of the embedded system, the effect of parasitic capaci- tance decreases the operating frequency range of the sensor in comparison to the measurements with the impedance analyzer as can be seen in Fig. 3.

For the quality evaluation of embedded system design, the standard deviation using C-75 metal was calculated. The 10 measurements were taken for the value of inductance and the standard deviation was calculated out of these measurements. The maximum standard deviation for unhardened and hardened $\mathrm{C} 75$ steel is $0.013 \mu \mathrm{H}$ at $20 \mathrm{kHz}$.

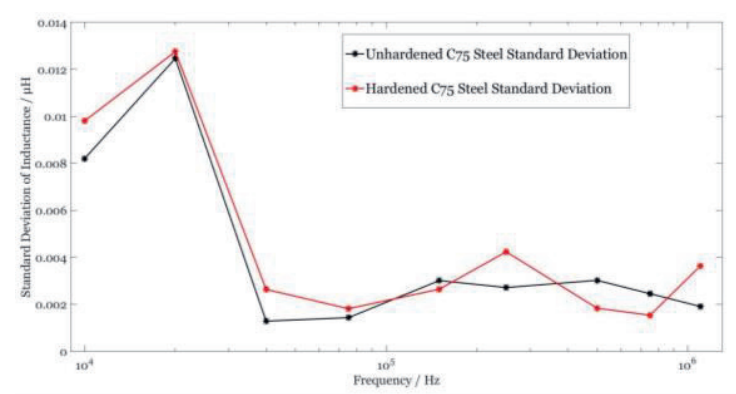

Fig. 4. Standard Deviation of Measurement System

\section{Conclusion}

The presented embedded measurement system could successfully retrieve broadband impedance data from the sensor coil. This impedance data was used to calculate the spectra of the inductance. In comparison to the model of Dodd \& Deeds, the measured inductance spectra display the same characteristic drop of inductance as predicted in the model. A more detailed comparison with a commercial impedance analyzer confirms this characteristic behavior. In the direct comparison to the impedance analyzer data, the embedded system has a characteristic inductance offset and a reduced frequency range due to the resonance of the sensor coil. Both effects are accounted to the parasitic inductance and capacitance on the PCB of the embedded system. A calibration procedure and adjustment of those systematic errors might improve the obtained inductance spectra in the future.

\section{References}

[1] R. Munjal, F. Wendler and O. Kanoun, "Embedded Wideband Measurement System for Fast Impedance Spectroscopy Using Undersampling," in IEEE Transactions on Instrumentation and Measurement, vol. 69, no. 6, pp. 3461-3469, 2020.

[2] R. Munjal et al., Effect of hardening on electrical and magnetic properties of C-75 steel and characterization with multi-frequency inductance spectroscopy, Measurement Science and Technology, vol. 32, no. 2, 2020.

[3] C. Dodd and W. Deeds, Analytical Solutions to EddyCurrent Probe-Coil Problems, Journal of Applied Physics, vol. 39, no. 6, pp. 2829-2838, 1968. 